\title{
Structure of Magnetic Noise in Dynamic Fracture
}

\author{
F. Kun, ${ }^{1}$ Gy. B. Lenkey, ${ }^{2}$ N. Takács, ${ }^{3}$ and D. L. Beke ${ }^{4}$ \\ ${ }^{1}$ Department of Theoretical Physics, University of Debrecen, P.O. Box: 5, H-4010 Debrecen, Hungary \\ ${ }^{2}$ Bay Zoltán Foundation for Applied Research, Institute for Logistics and Production Systems, Miskolc, Hungary, \\ ${ }^{3}$ Metalelektro Ltd. Borszék köz 13, H-1119 Budapest, Hungary \\ ${ }^{4}$ Department of Solid State Physics, University of Debrecen, P. O. Box:2, H-4010 Debrecen, Hungary
}

(Received 12 March 2004; published 23 November 2004)

\begin{abstract}
We present an experimental study of magnetic emission spectra recorded during impact fracture of steel. Novel features of dynamic fracture are revealed, i.e., the distribution of the voltage signals of the spectra; furthermore, the areas and energies of voltage peaks exhibit a power law behavior. The value of the exponents of the distributions proved to be characteristic for the failure mode: ductile failure gives rise to exponents significantly higher than brittle failure. The results imply that magnetic crackling noise accompanying impact fracture has a scale invariant structure which reveals new aspects of the dynamics of the fracture process.
\end{abstract}

DOI: 10.1103/PhysRevLett.93.227204

Crackling noise arises in a system if it responds to a continuous external driving in the form of discrete, impulsive bursts of size spanning orders of magnitude. This jerky response is abundant in nature, it has been observed in a large diversity of systems such as ferromagnetic materials [1-5], plastically deforming crystals [6], superconductors [7], and stressed solids [8-14]. Very recently, the Barkhausen noise arising in ferromagnetic materials under driving by a slowly varying magnetic field [1-5], and the acoustic emission of disordered solids subjected to a quasistatic loading have been intensively studied [8-14]. From the theoretical point of view it is of high importance to understand the statistical properties of crackling noise spectra recorded as a series of pulse signals. In diverse systems the distribution of crackling amplitudes and durations have been found to follow a universal power law form over a broad range [1-4,6-14]. The observed universality has been theoretically explained in terms of critical phenomena and self-organized criticality $[1,2,11,13,14]$. Besides its theoretical importance, the analysis of crackling noise has gained technical applications; by now it is a widely used diagnostic tool in nondestructive test methods.

In technical applications and engineering practice structural components are frequently exposed to dynamic loading so that their strength and reliability under dynamic loading conditions are of outstanding importance. While quasistatic fracture of disordered materials evolves by random nucleation of microcracks and the system attains equilibrium after each breaking event [8-14], in dynamic fracture a single crack emerges which proceeds at a high speed [15-18]. Recently, several interesting features of dynamic fracture have been revealed concerning the dynamics of crack propagation, the effect of heterogeneities of the underlying material, the evolution of roughness of the crack front and of the crack surface left behind [16]. The dynamic fracture of ferromagnetic materials like steel is accompanied by sudden changes of
PACS numbers: 75.60.Ej, 46.50.+a, 62.20.Mk, 75.60.Ch

the magnetic field which can be observed by a magnetic transducer. The transducer's output voltage is the magnetic emission (ME) signal which is proportional to the induced flux of the magnetic field. ME measurements complete traditional testings on dynamic fracture and provide additional insight into the fracture process. In recent experiments ME spectra have been found to show a hierarchy of peaks which addresses the possibility of structured crackling noise in impact fracture similarly to other driven disordered systems [19].

In this Letter we present a detailed study of the structure of ME spectra recorded in dynamic fracture of steel specimens for the first time in the literature. It is found that the distribution of voltages, furthermore, of the peak areas and energies exhibit a power law behavior in the regime dominated by crack propagation. The comparison of specimens of brittle and ductile failure showed that the value of the exponents of the power law decay is characteristic for the failure mode: ductile failure results in exponents significantly higher than brittle failure which is the consequence of the different dynamics of the cracking process. The results imply that magnetic crackling noise accompanying dynamic fracture has a scale invariant structure. The clear dependence of the exponents on the failure mode can be exploited for material's testing.

In dynamic fracture testing the so-called instrumented Charpy impact test is a widely used method to determine characteristic quantities like the dynamic fracture toughness of specimens [19]. In this test the load is exerted by a heavy pendulum which is raised to an initial height and released, striking and breaking the specimen at the bottom of its swing. The two ends of the specimen are clamped and the pendulum hits it in the middle resulting in a three point bending with a single growing crack [19]. The hammer of the pendulum is equipped with a pickup coil to record the ME signals. Our experiments have been carried out by means of an instrumented Charpy impact 
test machine on ferritic steel. For all the experiments the same material (E420-C high strength steel [19]) was used and the final breaking mode (brittle or ductile) was controlled by varying the temperature of the specimen between +20 and $-60{ }^{\circ} \mathrm{C}$. Two different impact velocities 2.75 and $5.5 \mathrm{~m} / \mathrm{s}$ were considered to obtain information about the effect of loading rate on ME spectra. For each experiment the force $F(t)$ exerted by the hammer on the specimen and the voltage signal generated in the pickup coil $V(t)$ were recorded as a function of time with a sampling rate of $10^{-6} \mathrm{~s}$. The force-displacement diagram $F(s)$, i.e., the constitutive behavior of the specimen can be obtained from the measured $F(t)$ by integration, first determining the velocity of the pendulum $v(t)=$ $v_{0}-\frac{1}{m} \int_{0}^{t} F\left(t^{\prime}\right) d t^{\prime}$, then the displacement follows as $s(t)=\int_{0}^{t} v\left(t^{\prime}\right) d t^{\prime}$. Here $m$ denotes the mass of the hammer and $v_{0}$ is its velocity at the instant of impact. A typical example of the force-time diagram obtained by instrumented impact testing is presented in Fig. 1 for a specimen of brittle and ductile failure.

In Fig. 1 the brittle or ductile nature of failure can be unambiguously determined from the behavior of $F(t)$ since brittle failure occurs in the form of a catastrophic event accompanied by a sudden drop of the force signal. When the failure is ductile or stable crack propagation precedes the unstable one, the specimen undergoes a large deformation and $F(t)$ develops a broad plateau from which even the instant of crack initiation is hard to obtain (see Fig. 1).

In spite of the relatively smooth force signal the corresponding magnetic emission spectra recorded in dynamic fracture experiments on ferromagnetic materials are composed of more or less well separated voltage peaks similarly to magnetically induced Barkhausen signals (see Fig. 2). In both brittle and ductile cases at the instant of hit a high peak occurs which is then followed by a sequence of peaks of fluctuating height until the duration of contact of the sample and the hammer of the impact machine. Two major microscopic mechanisms contribute to the voltage peaks: mechanically induced Barkhausen noise occurs due to the sudden changes of

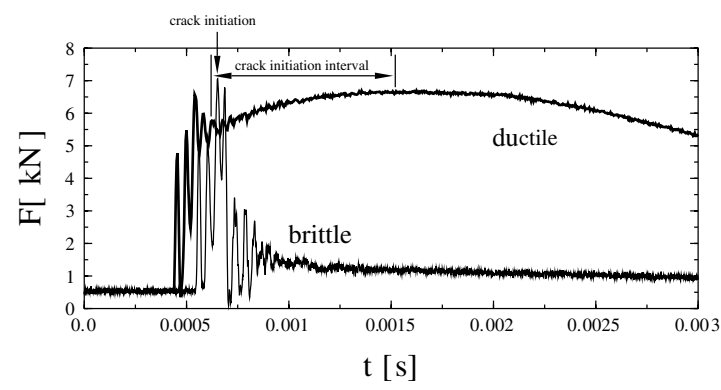

FIG. 1. Force-time histories for a sample of brittle and ductile failure obtained at $v_{0}=2.75 \mathrm{~m} / \mathrm{s}$ impact velocity. For brittle failure the instant of crack initiation corresponds to the maximum of $F(t)$ indicated by an arrow, however, in the ductile case only a time interval can be specified. the domain structure of the material under external mechanical loading.

This mechanism determines the magnetic emission spectrum before crack initiation and typically results in low peaks. After crack initiation the opening of the growing crack causes the magnetic field to penetrate from the solid to the gap between crack surfaces resulting in changes of the external magnetic field. This mechanism provides the dominating contribution for the voltage signals after crack initiation and is responsible for the higher peaks of the spectrum. It is interesting to note that even visual inspection reveals differences in Fig. 2 between the structure of the ME spectra emerging in different failure modes, i.e., ductile failure seems to give rise to broader peaks with smaller height than the brittle one. In order to quantify the structural properties of ME spectra we apply the techniques developed earlier for the study of magnetically induced Barkhausen noise [3,4].

A background voltage level $b_{l}$ is set to that horizontal cut of $V(t)$ which provides the maximum number of intersections, i.e., $b_{l}$ is the most probable voltage value of the spectrum. A peak of the spectrum is identified as that part of the voltage-time $V(t)$ curve which falls between two consecutive intersections with the background level at time values $t_{i}$ and $t_{i+1}$. A peak of index $i$ is characterized by its duration (width) $T_{i}=t_{i+1}-t_{i}$, area $A_{i}=\int_{t_{i}}^{t_{i+1}}\left[V(t)-b_{l}\right] d t$, height $h_{i}=\max V(t)$ for $t_{i}<t<$ $t_{i+1}$, and energy $E_{i}=\int_{t_{i}}^{t_{i+1}}\left[V(t)-b_{l}\right]^{2} d t$. In the present study we analyze the distribution of voltages $V$ of the ME spectra along with the area and energy distributions of peaks. The entire analysis is restricted to the duration of contact of the hammer and the specimen, which can be unambiguously determined from the force-time diagram, together with the failure mode. Since brittle failure gives rise to a short train of voltage signals, results on brittle fracture generally have worst statistics than the ductile ones: on the average, the ME spectrum of brittle specimens is composed of 1800 voltage values, in which the

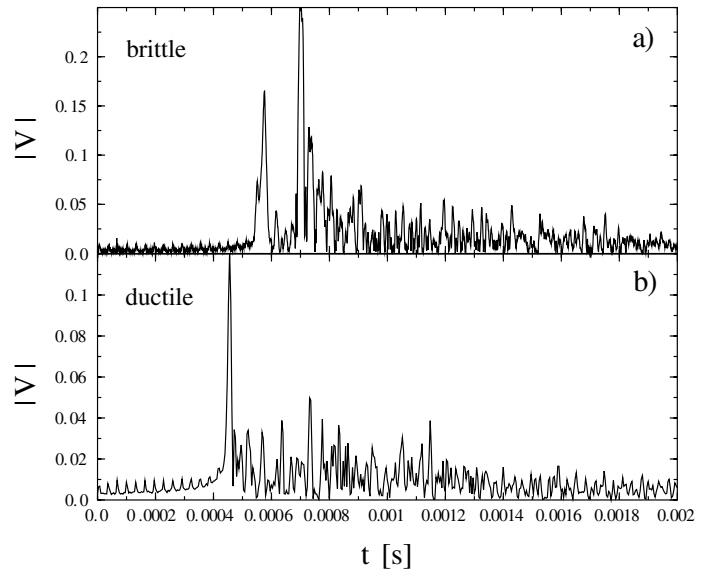

FIG. 2. Magnetic emission spectra $|V(t)|$ for a sample of brittle and ductile failure corresponding to the force-time diagrams of Fig. 1. 
data analyses algorithm identifies $80-100$ peaks, independently of the impact speed. In the case of ductile failure the average number of voltages is about 4000 and 3000, which give rise to 450 and 220 peaks at the impact velocities 2.75 and $5.5 \mathrm{~m} / \mathrm{s}$, respectively.

The distribution of voltages $p(V)$ of the ME spectra is presented in Fig. 3 for two different impact velocities averaging over ten (ductile) and eight (brittle) samples of the same type of failure obtained under identical conditions.

In all cases $p(V)$ is composed of two distinct regimes: for large voltage values, characteristic for crack propagation and opening, the distributions show a power law behavior $p(V) \sim V^{-\beta}$ over a range of about 1 order of magnitude, while for lower voltages, typical for deformation induced Barkhausen noise and artificial instrumental noise, no definite functional form can be deduced.

Voltages of the power law regime are determined by the high peaks of the spectrum, which are the fingerprints of the jerky extension and opening of the crack. The highest peaks of the spectra emerge at the initial jump of the crack at the onset time, which provides a very poor statistics in the vicinity of the cutoff of the distributions (see Figs. 2 and 3). The analysis of specimens of brittle and ductile failure showed that the overall form of the distribution is always the same, however, the value of the exponent of the power law regime is characteristic for the failure mode: ductile failure is characterized by an exponent $\beta=3.15 \pm 0.15$ significantly higher than brittle failure $\beta=2.2 \pm 0.2$. The larger $\beta$ of ductile failure indicates that large voltages, higher peaks, occur less frequently than in the brittle case, due to the less erratic way of crack extension. Varying the impact velocity results solely in shift of the distribution, i.e., at lower impact velocity the voltage signals of the ME spectrum are lower but the shape of the distribution, the value of the exponent of the power law regime, and hence, the separation of failure modes do not change.

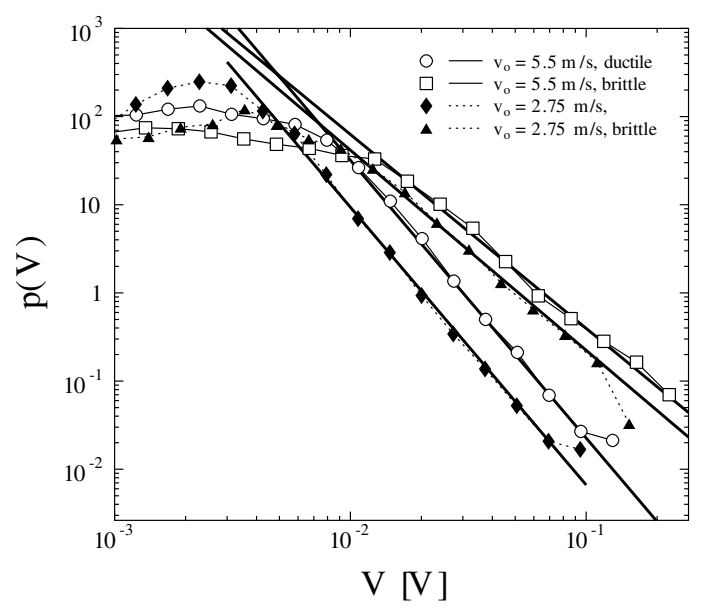

FIG. 3. Distribution of voltages $p(V)$ of ME spectra. The fitted straight lines are parallel for the same failure modes.
The peak area $A$, obtained as an integral of $V(t)$, is proportional to the change of the magnetic field under the peak duration, and hence is directly related to the extension of crack. In Fig. 4 the distribution of the peak areas $p(A)$ has an overall character similar to the voltage distribution $p(V)$, i.e., no definite shape for small peaks and a power law decay $p(A) \sim A^{-\tau}$ for larger peak areas. Contrary to the voltages $V$, the quantities characterizing peaks of the spectra reveal more information on the dynamics of the fracture process. Since under impact loading brittle failure occurs in the form of unstable crack propagation, the corresponding area distributions at different impact velocities only differ in the vicinity of the cutoff due to the sensitivity of the initial crack jump to the hitting speed $v_{0}$. The properties of stable crack propagation characterizing ductile failure are, however, strongly influenced by the impact speed $v_{0}$, i.e., at higher impact velocity peaks of larger area more often occur, which results in a slower decay of the distribution with a smaller value of $\tau$.

The value of the exponent $\tau$ of $P(A)$ is characteristic for the failure mode: $\tau=1.2 \pm 0.25$ was obtained for brittle failure, while $\tau=2.05 \pm 0.15$ and $\tau=1.85 \pm 0.18$ were determined for $v_{0}=2.75 \mathrm{~m} / \mathrm{s}$ and $v_{0}=5.5 \mathrm{~m} / \mathrm{s}$ in the ductile case, respectively.

The energy of peaks can be considered to be proportional to the energy dissipated by the crack extension under the peak duration. For both brittle and ductile failure $P(E)$ was found to follow a power law decay $P(E) \sim$ $E^{-\gamma}$, with substantial differences in the dependence on the impact velocity $v_{0}$. Because of the catastrophic nature of brittle fracture, the corresponding peak energy distributions do not exhibit any dependence on $v_{0}$. Except for the vicinity of the cutoff energy determined by the initial jump of the crack, the two curves of different impact velocities fall practically on the top of each other with an exponent $\gamma=1.15 \pm 0.2$; see Fig. 5. Ductile fracture proceeds in a long series of elementary steps giving rise to magnetic signals whose energy strongly depends on the

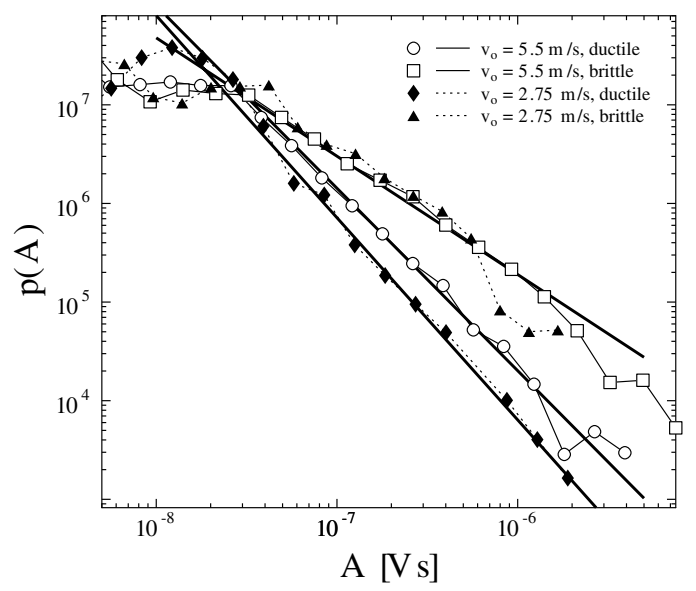

FIG. 4. Area distribution of peaks $p(A)$. In the brittle case the straight line was fitted to the data at $v_{0}=5.5 \mathrm{~m} / \mathrm{s}$. 


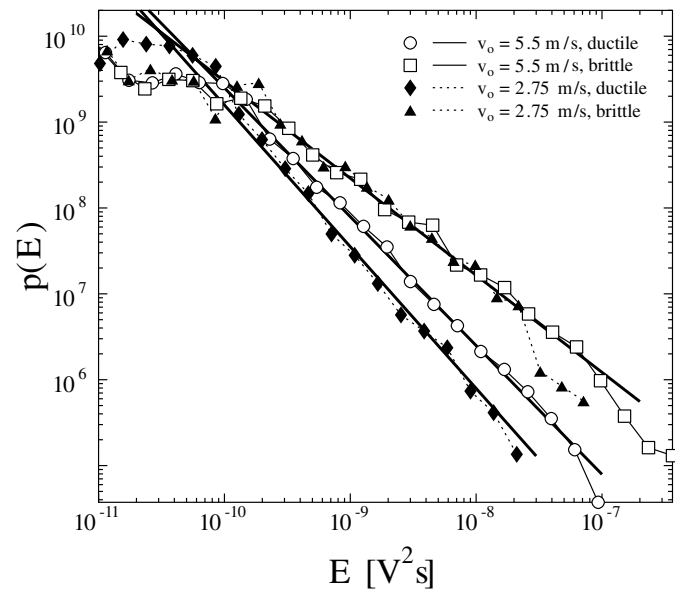

FIG. 5. Distribution of peak energies $p(E)$.

impact velocity $v_{0}$. At higher $v_{0}$ the crack propagates faster resulting in a smaller number of peaks, however, with a larger fraction of peaks of higher energy. This mechanism lowers the exponent of the power law regime at higher impact velocities: $\gamma=1.65 \pm 0.15$ and $\gamma=$ $1.45 \pm 0.15$ were obtained for $v_{0}=2.75 \mathrm{~m} / \mathrm{s}$ and $v_{0}=$ $5.5 \mathrm{~m} / \mathrm{s}$, respectively. It can be seen in Fig. 1 that before reaching the maximum, $F(t)$ shows 3-4 oscillations which are the consequence of elastic waves running through the sample. This implies that during the impact process the system is more or less equilibrated (the socalled $3 \tau$ condition of dynamic fracture is satisfied [19]) at the velocities considered which enables us to make comparison to recent theoretical findings. Lattice models of dynamic fracture composed of springs or beams provide a direct access to the damage on the microlevel and are able to relate the cracking to the macroscopic noisy response of the material [16-18,20,21]. In the continuous damage model [20] a power law distribution of burst energies was found under strain controlled loading along the plastic plateau where localization and crack growth dominates the damage process. The exponent $\gamma=1.2 \pm$ 0.1 was obtained in the limit of slow driving. In recent simulations of strain driven dynamic fracture of a lattice of springs the distribution of the energy bursts proved to be a power law of an exponent $\gamma=1.7 \pm 0.1$ independent on the loading rate [21]. Our experimental results fall between the bounds of the two simulation studies indicating a reasonable agreement.

In summary, our analysis revealed that quantities characterizing the structure of magnetic emission spectra recorded in dynamic fracture of ferromagnetic materials have a power law decay over a broad range, which is the fingerprint of scale free bursting activity accompanying the propagation and opening of the crack. Brittle failure occurs as a short, sudden event, while the power law distributed quantities in the ductile case characterize the regime of stable crack propagation where the system experiences a practically smooth driving similarly to other crackling systems [1-11]. The low statistics in the vicinity of the cutoff of the distributions is more or less an inherent handicap of this type of impact testing. A very interesting outcome of our analysis is the clear quantitative difference of the structure of ME spectra of brittle and ductile fracture behavior: the voltage signals and the peak area and energy distributions of ductile fracture show faster decay with higher exponents than the brittle ones, which is the consequence of the less erratic crack propagation in ductile failure. This feature can be exploited for materials testing and might open new possibilities for the precise determination of dynamic fracture characteristics of ferromagnetic materials, which are of high technological importance. The dependence of the exponents of ductile fracture on the impact velocity reveals changes of the crack dynamics with the driving rate.

This work was supported by the Projects NKFP No. 3/ 064/2001, NKFP-3A/043/04, OTKA T037212, T030057, and M041537. F. K. was supported by FKFP 0118/2001 and by the Gy. Békési Foundation of the HAS.

[1] G. Durin and S. Zapperi, Phys. Rev. Lett. 84, 4705 (2000).

[2] G. Durin and S. Zapperi, J. Appl. Phys. 85, 5196 (1999).

[3] G. Durin, A. Magni, and G. Bertotti, Fractals 3, 351 (1995).

[4] D. Spasojevic, S. Bukvic, S. Milosevic, and H. E. Stanley, Phys. Rev. E 54, 2531 (1996).

[5] R. A. White and K. A. Dahmen, Phys. Rev. Lett. 91, 085702 (2003).

[6] M. C. Miguel, A. Vespignani, S. Zapperi, J. Weiss, and J. Grasso, Nature (London) 410, 667 (2001).

[7] S. Field, J. Witt, F. Nori, and X. Ling, Phys. Rev. Lett. 74, 1206 (1995).

[8] A. Maes, C. Van Moffaert, H. Frederix, and H. Strauven, Phys. Rev. B 57, 4987 (1998).

[9] A. Guarino, A. Garcimartin, and S. Ciliberto, Eur. Phys. J. B 6, 13 (1998).

[10] A. Guarino, S. Ciliberto, A. Garcimartín, M. Zei, and R. Scorretti, Eur. Phys. J. B 26, 141 (2002).

[11] A. Petri, A. Paparo, A. Vespignani, A. Alippi, and M. Constantini, Phys. Rev. Lett. 73, 3423 (1994).

[12] A. Vespignani, A. Petri, A. Alippi, and G. Paparo, Fractals 3, 839 (1995).

[13] L. I. Salminen, A. I. Tolvanen, and M. J. Alava, Phys. Rev. Lett. 89, 185503 (2002).

[14] M. Kloster, A. Hansen, and P. C. Hemmer, Phys. Rev. E 56, 2615 (1997).

[15] M. Marder, Comput. Sci. Eng. 1, 2 (1999).

[16] J. Fineberg and M. Marder, Phys. Rep. 313, 1 (1999).

[17] M. Marder and X. Liu, Phys. Rev. Lett. 71, 2417 (1993).

[18] T. T. Rautiainen, M. J. Alava, and K. Kaski, Phys. Rev. E 51, 2727(R) (1994); 56, 6443 (1997).

[19] Gy. B. Lenkey and S. Winkler, Fatigue Fract. Eng. Mater. Struct. 20, 143 (1997).

[20] S. Zapperi, A. Vespignani, and H. E. Stanley, Nature (London) 388, 658 (1997).

[21] M. Minozzi, G. Caldarelli, L. Pietronero, and S. Zapperi, Eur. Phys. J. B 36, 203 (2003). 